\title{
ASYMPTOTIC ANALYSIS OF THE LINEARIZED NAVIER-STOKES EQUATION ON AN EXTERIOR CIRCULAR DOMAIN: EXPLICIT SOLUTION AND THE ZERO VISCOSITY LIMIT
}

\author{
Maria Carmela Lombardo, Russel E. Caflisch \& Marco Sammartino
}

To cite this article: Maria Carmela Lombardo, Russel E. Caflisch \& Marco Sammartino (2001) ASYMPTOTIC ANALYSIS OF THE LINEARIZED NAVIER-STOKES EQUATION ON AN EXTERIOR CIRCULAR DOMAIN: EXPLICIT SOLUTION AND THE ZERO VISCOSITY LIMIT, Communications in Partial Differential Equations, 26:1-2, 335-354, DOI: 10.1081/PDE-100001758

To link to this article: http://dx.doi.org/10.1081/PDE-100001758

曲 Published online: 07 Feb 2007.

Submit your article to this journal

Џll Article views: 48

Q View related articles $\sqsubset$

Citing articles: 6 View citing articles 


\title{
ASYMPTOTIC ANALYSIS OF THE LINEARIZED NAVIER-STOKES EQUATION ON AN EXTERIOR CIRCULAR DOMAIN: EXPLICIT SOLUTION AND THE ZERO VISCOSITY LIMIT
}

\author{
Maria Carmela Lombardo, ${ }^{1, \dagger}$ Russel E. Caflisch, $, 2, *$ \\ and Marco Sammartino ${ }^{1, \ddagger}$ \\ ${ }^{1}$ Dipartimento di Matematica, Università di Palermo, \\ Via Archirafi 34, 90123 Palermo, Italy \\ ${ }^{2}$ Department of Mathematics, University of California, \\ Los Angeles, USA
}

\begin{abstract}
In this paper we study and derive explicit formulas for the linearized Navier-Stokes equations on an exterior circular domain in space dimension two. Through an explicit construction, the solution is decomposed into an inviscid solution, a boundary layer solution and a corrector. Bounds on these solutions are given, in the appropriate Sobolev spaces, in terms of the norms of the initial and boundary data. The correction term is shown to be of the same order of magnitude as the square root of the viscosity.
\end{abstract}

Key Words: Navier-Stokes equations; Stokes equations; Boundary layer; Zero viscosity; Asymptotic analysis; Explicit solutions.

${ }^{*}$ Corresponding author. E-mail: caflisch@math.ucla.edu

†E-mail: lombardo@dipmat.math.unipa.it

†E-mail: marco@dipmat.math.unipa.it 


\section{INTRODUCTION}

In this paper we shall investigate the time dependent incompressible Stokes equations on an exterior circular domain in space dimension two, i.e.

$$
\begin{aligned}
\partial_{t} u_{\phi}+r^{-1} \partial_{\phi} p & =v\left(\Delta u_{\phi}-r^{-2} u_{\phi}+2 r^{-2} \partial_{\phi} u_{r}\right), \\
\partial_{t} u_{r}+\partial_{r} p & =v\left(\Delta u_{r}-r^{-2} u_{r}-2 r^{-2} \partial_{\phi} u_{\phi}\right), \\
\nabla \cdot \boldsymbol{u} & =0, \\
\gamma_{R} \boldsymbol{u} & =\boldsymbol{g}, \\
\boldsymbol{u}(r, \phi, t=0) & =\boldsymbol{u}_{0}(r, \phi),
\end{aligned}
$$

where $\boldsymbol{u}=\left(u_{r}(r, \phi, t), u_{\phi}(r, \phi, t)\right)$ is the velocity field, which depends on the radial variable $r$ with $r \geq R$, on the angular variable $\phi$ with $0 \leq \phi \leq 2 \pi$ and on the time variable $t \geq 0$. In the Equations (1)-(5) $\Delta=r^{-1} \partial_{r}\left(r \partial_{r}\right)+r^{-2} \partial_{\phi \phi}, \nabla \cdot \boldsymbol{u}=$ $r^{-1} \partial_{r}\left(r u_{r}\right)+r^{-1} \partial_{\phi} u_{\phi}, p=p(r, \phi, t)$ is the pressure, $v=\varepsilon^{2}$ is the viscosity coefficient and $\gamma_{R}$ is the trace operator defined by $\gamma_{R} f(r, \phi, t)=f(R, \phi, t)$. Equations (1) and (2) are the conservation of momentum for a viscous fluid obtained by linearizing the Navier-Stokes equation, Equation (3) is the incompressibility condition and Equations (4) and (5) are the boundary and the initial conditions, respectively. We are interested in the behavior of the solution in the limit of small viscosity. In fact the problem of the convergence of the Navier-Stokes equations to the Euler equation in presence of boundaries is a relevant problem in fluid dynamics, and Stokes equations can be considered a good simplified mathematical model that can be helpful in the understanding of the more complicated nonlinear case. When the Reynolds number is large the fluid shows two different regimes: far away from the boundary the viscous forces are negligible with respect to the inertial forces and the behavior of the fluid is believed to be well described by the Euler equations. They are obtained neglecting the viscosity term. As a consequence of the change in the order of the equations, only the no-flux boundary condition can be imposed. In the vicinity of the boundary, on the other hand, the viscous forces are not negligible and a boundary layer whose thickness is proportional to the square root of the viscosity appears. The no-slip condition causes a rapid variation of the tangential component of the velocity to adjust the flow to the value given by the inviscid outer theory. In the boundary layer the fluid is ruled by the Prandtl equations, which are obtained rescaling the normal variable with the square root of the viscosity $(\varepsilon=\sqrt{v})$ and imposing that the derivative with respect to the rescaled variable of the tangential velocity is $O(1)$. Therefore, both Euler and Prandtl equations can be obtained from Navier-Stokes through formal asymptotic expansions.

In [1] it was proved that, for analytic solutions of the Navier-Stokes equations on the half space and for a short time, these approximations are indeed 
correct. The solution of Navier-Stokes equations was constructed as the sum of the Euler solution, the Prandtl solution and a correction which was proved to be vanishingly small with the square root of the viscosity. The initial data were restricted to be analytic. In this paper we shall be concerned with the incompressible Stokes equations on an exterior circular domain. Following the technique used by Ukai (see [2]) we will give an explicit formula for the solution and then we will use it to perform the asymptotic analysis in the limit of vanishing viscosity. We will show that the solution of the Stokes equations can be written in the form:

$$
\boldsymbol{u}^{\mathrm{S}}=\boldsymbol{u}^{\mathrm{E}}+\boldsymbol{u}^{\mathrm{P}}+\varepsilon \boldsymbol{w},
$$

where $\boldsymbol{u}^{\mathrm{E}}$ is the solution of the linearized Euler equations, $\boldsymbol{u}^{\mathrm{P}}$ is the Prandtl solution exponentially decaying outside the boundary layer, and $\varepsilon \boldsymbol{w}$ is the correction term. The explicit expression of all the terms of this expansion is given. This result is stated in Theorem 4.1, which is the main result of this paper. The paper is organized as follows: in Section 2 we shall introduce the spaces of functions we shall be using through the rest of this paper. In Section 3 we shall give the explicit formulas for the solution of the Stokes problem in the exterior circular domain. In Section 4 we shall perform the asymptotic analysis of the Stokes equations and give the appropriate estimates in terms of the initial and boundary data. In particular the norm of the term $\boldsymbol{w}$ in the expansion (6) is shown to be $O$ (1). In this paper all the estimates are given in the usual Sobolev spaces. Moreover the asymptotic expansion (6) is shown to be valid for an arbitrarily large time $T$.

\section{FUNCTION SPACES}

In this section we define some function spaces we shall be using through the rest of this paper. All functions depending on the angular variable $\phi$ will be periodic in this variable. We recall that the $L^{2}$ norm expressed in polar coordinates $(r, \phi)$ is weighted with $r$. We define the space $L_{r}^{2}$ as the space of those functions $f(r, \phi)$ such that

$$
\|f\|_{r}=\left\{\int_{0}^{2 \pi} \int_{R}^{\infty} \mathrm{d} \phi \mathrm{d} r r|f(r, \phi)|^{2}\right\}^{1 / 2}<\infty .
$$

We first introduce the ambient spaces for the inviscid equation.

Definition 2.1. $\quad H^{\prime l}$ is the set of all functions $f(\phi)$ such that

$$
\text { - } \partial_{\phi}^{j} f \in L^{2}([0,2 \pi]) \text { with } j \leq l \text {. }
$$

We shall denote the usual norm in $H^{\prime l}$ with $|f|_{l}$. 
Definition 2.2. $\quad H_{T}^{\prime l}$ is the set of all functions $f(\phi, t)$ such that

- $\partial_{t}^{j} f(\phi, t) \in L^{\infty}\left([0, T], H^{l-j}\right)$ with $j \leq l$.

The norm of $f \in H_{T}^{\prime l}$ is given by

$$
|f|_{l, T}=\sum_{j_{1}+j_{2} \leq l} \sup _{0 \leq t \leq T}\left\|\partial_{t}^{j_{1}} \partial_{\phi}^{j_{2}} f(\cdot, t)\right\|_{L^{2}([0,2 \pi])} .
$$

Definition 2.3. $\quad H^{l}$ is the set of all functions $f(r, \phi)$ defined on $\Omega=[R, \infty) \times$ $[0,2 \pi]$ such that

$$
\text { - } r^{-m} \partial_{\phi}^{m} \partial_{r}^{j} f \in L_{r}^{2} \text { with } m+j \leq l .
$$

The norm of $f$ is given by

$$
|f|_{l}=\sum_{m+j \leq l}\left\|r^{-m} \partial_{\phi}^{m} \partial_{r}^{j} f(\cdot, \cdot)\right\|_{r} .
$$

Definition 2.4. $\quad H_{T}^{l}$ is the set of all functions $f(r, \phi, t)$ such that

$$
\text { - } \partial_{t}^{j} f(r, \phi, t) \in L^{\infty}\left([0, T], H^{l-j}\right) \text { with } j \leq l .
$$

The norm of $f \in H_{T}^{l}$ is given by

$$
|f|_{l, T}=\sum_{j_{1}+j_{2}+j_{3} \leq l} \sup _{0 \leq t \leq T}\left\|r^{-j_{1}} \partial_{\phi}^{j_{1}} \partial_{t}^{j_{2}} \partial_{r}^{j_{3}} f(\cdot, \cdot, t)\right\|_{r} .
$$

We now introduce the ambient spaces for Pradtl equations. All the functions belonging to these spaces depend on the normal scaled variable $Y=\frac{r-R}{\varepsilon}$ and are exponentially decaying with respect to $Y$. We require differentiability with respect to this variable only up to the second order.

Definition 2.5. $K^{l, \mu}$ with $\mu>0$ is the set of all functions $f(Y, \phi)$ such that

- $\partial_{\phi}^{j_{1}} \partial_{Y}^{j_{2}} f(Y, \phi) \in L^{2}([0,2 \pi])$, with $j_{2} \leq 2$ and $j_{1}+j_{2} \leq l$, with $j_{1} \leq l-$ 2 if $j_{2}>0$.

- $\sup _{Y \geq 0} e^{\mu Y}\left\|\partial_{\phi}^{j_{1}} \partial_{Y}^{j_{2}} f(Y, \cdot)\right\|_{L^{2}}<\infty$ with $j_{2} \leq 2$ and $j_{1}+j_{2} \leq l$, with $j_{1} \leq$ $l-2$ if $j_{2}>0$.

The norm is given by

$$
\begin{aligned}
|f|_{l, \mu}= & \sum_{j \leq l} \sup _{Y \geq 0} e^{\mu Y}\left\|\partial_{\phi}^{j} f(Y, \cdot)\right\|_{L^{2}} \\
& +\sum_{0 \leq j_{2} \leq 2} \sum_{j_{1} \leq l-2} \sup _{Y \geq 0} e^{\mu Y}\left\|\partial_{\phi}^{j_{1}} \partial_{Y}^{j_{2}} f(Y, \cdot)\right\|_{L^{2}} .
\end{aligned}
$$


We now introduce the dependence on time. We require differentiability with respect to time only up to the first order. This is typical of parabolic type equations in presence of a boundary (see [3]).

Definition 2.6. $K_{T}^{l, \mu}$ with $\mu>0$ is the set of all functions $f(Y, \phi, t)$ such that

- $f \in L^{\infty}\left([0, T], K^{l, \mu}\right)$.

- $\partial_{t} \partial_{\phi}^{j} f \in L^{\infty}\left([0, T], K^{0, \mu}\right)$, with $j \leq l-2$.

The norm is given by

$$
\begin{aligned}
|f|_{l, \mu, T}= & \sum_{0<j_{2} \leq 2} \sum_{j_{1} \leq l-2} \sup _{0 \leq t \leq T} \sup _{Y \geq 0} e^{\mu Y}\left\|\partial_{\phi}^{j_{1}} \partial_{Y}^{j_{2}} f(Y, \cdot, t)\right\|_{L^{2}} \\
& +\sum_{j \leq l-2} \sup _{0 \leq t \leq T} \sup _{Y \geq 0} e^{\mu Y}\left\|\partial_{t} \partial_{\phi}^{j} f(Y, \cdot, t)\right\|_{L^{2}} .
\end{aligned}
$$

We now introduce the ambient spaces for the error equation. The following space is the natural ambient space for the first order correction term. All the functions belonging to $N_{T}^{l}$ are $L^{2}$ with respect to both tangential and normal variable; moreover only first order derivatives with respect to the time variable are allowed.

Definition 2.7. $\quad N_{T}^{l}$ with is the set of all functions $f(r, \phi, t)$ such that

- $r^{-j_{1}} \partial_{\phi}^{j_{1}} \partial_{r}^{j_{2}} f \in L^{\infty}\left([0, T], L_{r}^{2}\right)$ with $j_{1}+j_{2} \leq l$.

- $r^{-j} \partial_{\phi}^{j} \partial_{t} f \in L^{\infty}\left([0, T], L_{r}^{2}\right)$ with $j \leq l-2$.

The norm of $f \in N_{T}^{l}$ is given by

$$
\begin{aligned}
\|f\|_{l, T}^{N}= & \sum_{0<j_{2} \leq 2} \sum_{j_{1} \leq l-2} \sup _{0 \leq t \leq T}\left\|r^{-j_{1}} \partial_{\phi}^{j_{1}} \partial_{r}^{j_{2}} f(\cdot, \cdot, t)\right\|_{r} \\
& +\sum_{j \leq l-2} \sup _{0 \leq t \leq T}\left\|r^{-j} \partial_{\phi}^{j} \partial_{t} f(\cdot, \cdot, t)\right\|_{r} .
\end{aligned}
$$

We now introduce the ambient spaces for the overall correction term. All functions belonging to the following spaces are functions $L^{2}$ with respect to both tangential and normal variables. Notice that, due to the presence in the error equation of the rapidly varying terms arising from the Prandtl solution, the solution of the error equation will have a fast dependence on $r$. Therefore, in the following spaces, all the derivatives of order $j$ with respect to $r$ and of order $m$ with respect to $\phi$ are weighted with $\varepsilon^{j+m-1}$. 
Definition 2.8. $\quad L^{l}$ is the set of the functions $f(r, \phi)$ such that

- $\varepsilon^{j_{1}+j_{2}-1} r^{-j_{1}} \partial_{\phi}^{j_{1}} \partial_{r}^{j_{2}} f \in L_{r}^{2}$ with $j_{2} \leq 2$ and $j_{1}+j_{2} \leq l$, with $j_{1} \leq l-$ 2 if $j_{2}>0$.

The norm of $f \in L^{l}$ is given by

$$
\|f\|_{l}=\sum_{j_{1} \leq l}\left\|\varepsilon^{j_{1}-1} r^{-j_{1}} \partial_{\phi}^{j_{1}} f\right\|_{r}+\sum_{0<j_{2} \leq 2} \sum_{j_{1} \leq l-2}\left\|\varepsilon^{j_{1}+j_{2}-1} r^{-j_{1}} \partial_{\phi}^{j_{1}} \partial_{r}^{j_{2}} f\right\|_{r} .
$$

Definition 2.9. $\quad L_{T}^{\prime l}$ is the set of all functions $f(\phi, t)$ such that

- $\partial_{\phi}^{j} f \in L^{\infty}\left([0, T], H^{\prime 0}\right)$ with $j \leq l$.

- $\partial_{\phi}^{j} \partial_{t} f \in L^{\infty}\left([0, T], H^{\prime 0}\right)$ with $j \leq l-2$.

The norm of $f \in L_{T}^{\prime l}$ is given by

$$
\|f\|_{l, T}=\sum_{j \leq l} \sup _{0 \leq t \leq T}\left\|\partial_{\phi}^{j} f(\cdot, t)\right\|_{L^{2}}+\sum_{j \leq l-2} \sup _{0 \leq t \leq T}\left\|\partial_{\phi}^{j} \partial_{t} f(\cdot, t)\right\|_{L^{2}} .
$$

Definition 2.10. $L_{T}^{l}$ is the set of all functions $f(r, \phi, t)$ such that

- $f \in L^{\infty}\left([0, T], L^{l}\right)$.

- $\varepsilon^{j} r^{-j} \partial_{\phi}^{j} \partial_{t} f \in L^{\infty}\left([0, T], L^{0}\right)$ with $j \leq l-2$.

The norm of $f \in L_{T}^{l}$ is given by

$$
\begin{aligned}
\|f\|_{l, T}= & \sum_{0<j_{2} \leq 2} \sum_{j_{1} \leq l-2} \sup _{0 \leq t \leq T}\left\|\varepsilon^{j_{1}+j_{2}-1} r^{-j_{1}} \partial_{\phi}^{j_{1}} \partial_{r}^{j_{2}} f(\cdot, \cdot, t)\right\|_{r} \\
& +\sum_{j \leq l-2} \sup _{0 \leq t \leq T}\left\|\varepsilon^{j-1} r^{-j} \partial_{\phi}^{j} \partial_{t} f(\cdot, \cdot, t)\right\|_{r} .
\end{aligned}
$$

\section{THE STOKES EQUATIONS: EXPLICIT FORMULAS}

In this section we will give an explicit formula for the solution of the Stokes equation. In Subsection 3.1 we shall introduce some notation and a pseudodifferential operator that will be used in the last step of the solution of the Stokes systems. In Subsection 3.2 we will introduce the Weber transform and some of its properties. In Subsection 3.3 the Stokes equations are considered and the explicit solution is given through the Weber transform. Estimates are also given. 


\subsection{The Ukai Operator}

We will consider the Fourier expansion of $f(\phi)$ :

$$
f(\phi)=\sum_{-\infty}^{+\infty} \mathrm{e}^{i k \phi} \hat{f}(k) \text { where } \hat{f}(k)=\int_{0}^{2 \pi} \mathrm{e}^{-i k \phi} f(\phi) \mathrm{d} \phi .
$$

In what follows we shall adopt the convention that $k$ is the dual variable of the variable $\phi$.

If $T$ is an operator acting on a function $f(\phi)$ such that

$$
\widehat{T f}(k)=\sigma(T)(k) \hat{f}(k),
$$

then $\sigma(T)$ is called the symbol of the pseudodifferential operator $T$. With an abuse of notation we shall adopt the convention of omitting the distinction between a function and its Fourier coefficient and between an operator and its symbol.

We introduce the operator $U[\cdot, \cdot]$ that acts on functions $f(r, k)$ with $r \in$ $[R, \infty)$ and $g(k)$ :

$$
U[f, g]=U_{b}(g)+U_{s}(f),
$$

where

$$
\begin{aligned}
U_{b}(g) & =\left(\frac{R}{r}\right)^{|k|+1} g(k) . \\
U_{s}(f) & =\frac{|k|}{r^{|k|+1}} \int_{R}^{r} \mathrm{~d} r^{\prime} r^{\prime|k|} f\left(r^{\prime}, k\right) .
\end{aligned}
$$

The operator $U[f, g]$ solves the following problem:

$$
\begin{aligned}
\left(r \partial_{r}+|k|+1\right) U[f, g] & =|k| f, \\
\gamma_{R} U[f, g] & =g(k) .
\end{aligned}
$$

We now give an estimate on the above operators that will be useful in the sequel.

Proposition 3.1. Let $g \in H^{\prime l}$ and let $f \in L^{l}$. Then $U_{b}(g) \in H^{l}$ and $U_{s}(f) \in L^{l}$ and the following estimates hold:

$$
\left|U_{b}(g)\right|_{l} \leq|g|_{l}, \quad\left\|U_{s}(f)\right\|_{l} \leq\|f\|_{l} .
$$

Proof: The proof of the first estimate is obvious since $\left(\frac{R}{r}\right)^{|k|+1} \leq 1$. The second inequality can be easily proved in the $r$-norm through Jensen's inequality, following the same line of [4]. An alternative way, as suggested by the Referee, is to multiply Equation (19) by $r U$, integrating and using a weighted Cauchy-Schwartz estimate. In order to get the estimate in the $l$-norm one has to repeat the same steps as above for the function $\varepsilon^{j+m-1} r^{-m} \partial_{\phi}^{m} \partial_{r}^{j} f(r)$. Note that this proposition could have been 
stated for $f$ and $u_{s}(f)$ in $H_{j}^{\ell} ; L^{\ell}$ has been used because it is the natural space for the errors estimates below.

\subsection{The Weber Transform and the Heat Operators}

This subsection deals with the definition and the properties of the Weber transform which arises naturally in the discussion of axisymmetrical problems formulated in cylindrical polar coordinates (see [5]). Through the Weber transform we will solve the heat equation and define the heat operator that will be useful for the solution of the Stokes system.

Given $f(r)$ defined on $[R, \infty)$, the Weber transform of order $n$ is defined by the formula

$$
W_{n}\{f\}(p)=\int_{R}^{\infty} \mathrm{d} r r Z_{n}(p r) f(r),
$$

where

$$
Z_{n}(p r)=J_{n}(p r) Y_{n}(p R)-Y_{n}(p r) J_{n}(p R) .
$$

In the above expression $J_{n}(x)$ and $Y_{n}(x)$ are the Bessel functions of order $n$ of the first and the second kind, respectively and $Z_{n}(p r)$ are the cylinder functions satisfying the boundary condition $Z_{n}(p R)=0$. The inverse Weber transform is defined by

$$
f(r)=\int_{0}^{\infty} \mathrm{d} p p \frac{Z_{n}(p r)}{J_{n}^{2}(p R)+Y_{n}^{2}(p R)} W_{n}\{f\}(p) .
$$

The cylinder functions satisfy Bessel differential equation, namely

$$
\frac{\mathrm{d}}{\mathrm{d} r}\left[r \frac{\mathrm{d}}{\mathrm{d} r} Z_{n}(p r)\right]+r\left(p^{2}-\frac{n^{2}}{r^{2}}\right) Z_{n}(p r)=0
$$

In what follows we shall need the following lemma.

Lemma 3.1 (Bessel operator). If the Bessel operator of order $n$ is defined as

$$
B_{n}(f)=\left(\partial_{r r}+\frac{1}{r} \partial_{r}-\frac{n^{2}}{r^{2}}\right) f
$$

then

$$
W_{n}\left\{B_{n}(f)\right\}=\frac{2}{\pi} f(R)-p^{2} W_{n}\{f\}
$$

provided that both $r f^{\prime}(r)$ and $r f(r)$ decay as $r \rightarrow \infty$.

We are interested in solving the heat equation expressed in polar coordinates on an exterior circular domain. It is known that the initial boundary value problem for the heat equation is uniquely solvable in Sobolev spaces, provided that the 
initial and the boundary data satisfy the compatibility conditions (see [3]). Since we are interested in giving an explicit solution formula for the heat equations below, we will make use of the Weber transform, and we will refer to the cited bibliography for the estimates in the functional spaces.

Let us solve the heat equation with source term, boundary data, and initial data:

$$
\begin{aligned}
{\left[\partial_{t}-\varepsilon^{2}\left(\partial_{r r}+\frac{1}{r} \partial_{r}+\frac{1}{r^{2}} \partial_{\phi \phi}\right)\right] u } & =f(r, \phi, t), \\
\gamma_{R} u & =g(\phi, t), \\
u(r, \phi, t=0) & =u_{0}(r, \phi) .
\end{aligned}
$$

If we take the Fourier transform with respect to the $\phi$ variable, the Weber transform with respect to the $r$ variable and make use of the Lemma 3.1, we get the following ODE for $W_{k}\{u\}$ :

$$
\begin{aligned}
\left(\frac{\mathrm{d}}{\mathrm{d} t}+\varepsilon^{2} p^{2}\right) W_{k}\{u\}(p, k, t) & =\frac{2 \varepsilon^{2}}{\pi} g+W_{k}\{f\}, \\
W_{k}\{u\}(p, k, t=0) & =W_{k}\left\{u_{0}\right\} .
\end{aligned}
$$

If one solves the above system and takes the inverse Weber transform, then the solution of the system (24)-(26) can be written in the form

$$
u(r, \phi, t)=\mathcal{E}_{k}^{(2)} f+\mathcal{E}_{k}^{(1)} g+\mathcal{E}_{k}^{(0)} u_{0},
$$

where the explicit expressions of the above operators are given by

$$
\begin{aligned}
& \mathcal{E}_{k}^{(2)} f=W_{k}^{-1}\left\{\int_{0}^{t} \mathrm{~d} s \mathrm{e}^{-\varepsilon^{2} p^{2}(t-s)} W_{k}\{f\}\right\}, \quad \mathcal{E}_{k}^{(0)} u_{0}=W_{k}^{-1}\left\{\mathrm{e}^{-\varepsilon^{2} p^{2} t} W_{k}\left\{u_{0}\right\}\right\}, \\
& \mathcal{E}_{k}^{(1)} g=W_{k}^{-1}\left\{\int_{0}^{t} \mathrm{~d} s \mathrm{e}^{-\varepsilon^{2} p^{2}(t-s)} \frac{2 \varepsilon^{2}}{\pi} g\right\} .
\end{aligned}
$$

In the above expressions $\mathcal{E}_{k}^{(2)} f$ is the $k$ th Fourier coefficient of the operator which inverts the heat equation with homogeneous boundary and initial data, $\mathcal{E}_{k}^{(1)} g$ is the $k$ th Fourier coefficient of the operator which solves the homogeneous heat equation with nonzero boundary data and zero initial data and $\mathcal{E}_{k}^{(0)} u_{0}$ is the $k$ th Fourier coefficient of the operator which solves the homogeneous heat equation with zero boundary data and nonzero initial data.

We now give some estimates on the solution of the above heat equations.

Proposition 3.2. Let $f(r, \phi, t) \in L_{T}^{l}, u_{0}(r, \phi) \in L^{l}$ and $g(\phi, t) \in L_{T}^{\prime l}$. Let the compatibility condition $\gamma_{R} u_{0}=g(\phi, t=0)$ be satisfied. Then the solution $u$ of the heat equations (24)-(26) is in $L_{T}^{l}$ and the following estimate holds:

$$
\|u\|_{l, T} \leq c\left[\|f\|_{l, T}+\left\|u_{0}\right\|_{l}+|g|_{l, T}\right] .
$$


The proof of the above proposition can be achieved following the same steps as in [3] (see e.g., Chapter IV, Theorem 5.2, and Theorem 9.1). There are two main differences to be considered. The first one regards the fact that, in [3], the unique solvability of the linear parabolic Boundary-Value Problem is proved in function spaces where the same norm is considered with respect to space and time variable (either the sup-norm or the $L^{q}$-norm), while we consider the sup-norm for the time variable and the $L^{2}$-norm for the space variables. This is a merely formal difference since the norms in space and in time are independent from each other.

The second difference concerns the $\varepsilon$, which is absent in the equations of the cited reference. To apply such theorems to the solutions of Equations (24)-(26), it is enough to define the rescaled variable $r^{\prime}=\frac{r}{\varepsilon}$. Therefore the coefficients of the transformed heat equation do not depend on $\varepsilon$ and the estimates of [3] are directly applicable to Equations (24)-(26) in the space $L_{T}^{l}$ (where the normal derivatives are weighted with $\varepsilon$ ).

\subsection{The Stokes Equation}

In this subsection we consider the following equations:

$$
\begin{aligned}
& \partial_{t} u_{\phi}-\varepsilon^{2}\left[\left(\partial_{r r}+\frac{1}{r} \partial_{r}-\frac{k^{2}}{r^{2}}\right) u_{\phi}-r^{-2} u_{\phi}+\frac{2 i k}{r^{2}} u_{r}\right]+r^{-1} \partial_{\phi} p \\
& \quad=f_{\phi}(r, k, t), \\
& \partial_{t} u_{r}-\varepsilon^{2}\left[\left(\partial_{r r}+\frac{1}{r} \partial_{r}-\frac{k^{2}}{r^{2}}\right) u_{r}-r^{-2} u_{r}-\frac{2 i k}{r^{2}} u_{\phi}\right]+\partial_{r} p \\
& \quad=f_{r}(r, k, t), \\
& \nabla \cdot \boldsymbol{u}=0, \\
& \gamma_{R} \boldsymbol{u}=\boldsymbol{g}(k, t), \\
& \boldsymbol{u}(r, \phi, t=0)=\boldsymbol{u}_{0} .
\end{aligned}
$$

Taking the divergence of Equations (30) and (31) a straightforward calculation shows that the pressure $p$ is harmonic, i.e. using the Fourier transform:

$$
\Delta p=\left(\frac{1}{r} \partial_{r}-\frac{|k|}{r^{2}}\right)\left(r \partial_{r}+|k|\right) p=0 .
$$

Imposing the pressure to be finite at infinity we get

$$
\left(r \partial_{r}+|k|\right) p=0
$$

which also implies

$$
\left(r \partial_{r}+|k|+1\right) \partial_{r} p=\partial_{r}\left(r \partial_{r}+|k|\right) p=0 .
$$


On the other hand, using the incompressibility condition, Equation (31) becomes

$$
\partial_{t} u_{r}-\varepsilon^{2} \Delta_{M} u_{r}+\partial_{r} p=f_{r},
$$

where $\Delta_{M} \equiv\left(\partial r r+\frac{3}{r} \partial_{r}-\frac{k^{2}-1}{r^{2}}\right)$. It is easily seen that $\Delta_{M}$ can be written in the form

$$
\Delta_{M}=\left(\frac{1}{r} \partial_{r}-\frac{|k|-1}{r^{2}}\right)\left(r \partial_{r}+|k|+1\right) .
$$

Therefore, if we define $|k| \tau$ as

$$
|k| \tau=\left(r \partial_{r}+|k|+1\right) u_{r}
$$

and apply $\left(r \partial_{r}+|k|+1\right)$ to Equation (37), we get the following equation for $|k| \tau$ :

$$
\partial_{t}|k| \tau-\varepsilon^{2}\left(r \partial_{r}+|k|+1\right)\left(\frac{1}{r} \partial_{r}-\frac{|k|-1}{r^{2}}\right)|k| \tau=\left(r \partial_{r}+|k|+1\right) f_{r} .
$$

Notice that the pressure does not appear because of Equation (36). Moreover, since

$$
\left(r \partial_{r}+|k|+1\right)\left(\frac{1}{r} \partial_{r}-\frac{|k|-1}{r^{2}}\right)=\partial_{r r}+\frac{1}{r} \partial_{r}-\frac{(|k|-1)^{2}}{r^{2}}
$$

we get the following equation for $|k| \tau$ :

$$
\left[\partial_{t}-\varepsilon^{2}\left(\partial_{r r}+\frac{1}{r} \partial_{r}-\frac{(|k|-1)^{2}}{r^{2}}\right)\right]|k| \tau=\left(r \partial_{r}+|k|+1\right) f_{r} .
$$

The boundary and initial conditions read

$$
\begin{aligned}
\gamma|k| \tau & =\gamma\left(r \partial_{r}+|k|+1\right) u_{r}=\gamma\left(-i k u_{\phi}-u_{r}+(|k|+1) u_{r}\right) \\
& =|k| V_{1} \boldsymbol{g} \\
|k| \tau(t & =0)=|k| V_{1} \boldsymbol{u}_{0}
\end{aligned}
$$

where the operator $V_{1}$ is defined as

$$
V_{1} \boldsymbol{g}=g_{r}-N^{\prime} g_{\phi}
$$

and $N^{\prime}$ is defined as

$$
N^{\prime}=\frac{i k}{|k|} \text {. }
$$

Notice that, using the incompressibility condition for $\boldsymbol{f}$, the source term in Equation (39) can be expressed in terms of $V_{1}$ as $|k| V_{1} f$. Since the spatial operator on the left hand side of Equation (39) is the Bessel operator of order $|k|-1$, to solve Equations (39)-(41) we will make use of the Weber transform. Using the result given in Equation (29), one gets

$$
\tau(r, k, t)=\mathcal{E}_{|k|-1}^{(2)} V_{1} \boldsymbol{f}+\mathcal{E}_{|k|-1}^{(1)} V_{1} \boldsymbol{g}+\mathcal{E}_{|k|-1}^{(0)} V_{1} \boldsymbol{u}_{0} .
$$


Solving Equation (38) with the boundary condition (33) one gets for $u_{r}$

$$
u_{r}=U\left[\tau, g_{r}\right] \text {, }
$$

where $U$ is the operator defined in the Subsection 3.1. Through the incompressibility condition one gets $u_{\phi}$ :

$$
u_{\phi}=N^{\prime}\left[\tau(r, k, t)-u_{r}(r, k, t)\right] .
$$

Using the expressions (43) and (44) one can finally express the solution of the Stokes equations in the following form:

$$
\boldsymbol{u}=\boldsymbol{u}_{(b)}+\boldsymbol{u}_{(s)},
$$

where

$$
\begin{aligned}
& \boldsymbol{u}_{(b)}=\left(U_{b}\left(g_{r}\right),-N^{\prime} U_{b}\left(g_{r}\right)\right), \\
& \boldsymbol{u}_{(s)}=\left(U_{s}(\tau), N^{\prime}\left(\tau-U_{s}(\tau)\right)\right) .
\end{aligned}
$$

We now estimate the above solution. We first state the following lemma.

Lemma 3.2. Let $\boldsymbol{u}_{0} \in L^{l}, \boldsymbol{f} \in L_{T}^{l}$ and $\boldsymbol{g} \in L_{T}^{\prime l}$. Suppose the compatibility condition between the boundary and initial data $\gamma_{R} \boldsymbol{u}_{0}=\boldsymbol{g}(\phi, t=0)$ is satisfied. Then $\tau \in L_{T}^{l}$ and the following estimate holds:

$$
\|\tau\|_{l, T} \leq c\left(\left\|\boldsymbol{u}_{0}\right\|_{l}+\|\boldsymbol{f}\|_{l, T}+\|\boldsymbol{g}\|_{l, T}\right) .
$$

The proof of the above lemma is based on the fact that $\tau$ satisfies Equations (39)-(41), which are of the same form as Equations (24)-(26), with the boundary and initial data satisfying the compatibility conditions. Therefore Proposition 3.2 applies and the statement of the lemma follows. The main result of this section is the following proposition.

Proposition 3.3. Let $\boldsymbol{u}_{0} \in L^{l}$, and $\boldsymbol{f} \in L_{T}^{l}$ and $\boldsymbol{g} \in L_{T}^{\prime l}$. Suppose the compatibility condition between the boundary and initial data $\gamma_{R} \boldsymbol{u}_{0}=\boldsymbol{g}(\phi, t=0)$ is satisfied. Then the solution of Equations (30)-(34) $\boldsymbol{u}$ can be decomposed in the form (45), with $\boldsymbol{u}_{(b)} \in N_{T}^{l}, \boldsymbol{u}_{(s)} \in L_{T}^{l}$ and the following estimates hold:

$$
\begin{aligned}
\left\|\boldsymbol{u}_{(b)}\right\|_{l, T}^{N} & \leq\|g\|_{l, T} \\
\left\|\boldsymbol{u}_{(s)}\right\|_{l, T} & \leq c\left[\left\|\boldsymbol{u}_{0}\right\|_{l}+\|\boldsymbol{f}\|_{l, T}+\|\boldsymbol{g}\|_{l, T}\right] .
\end{aligned}
$$

The proof is easily achieved using the explicit expressions for $\boldsymbol{u}_{(b)}$ and $\boldsymbol{u}_{(s)}$ given in Equations (46) and (47), through the estimate on $\tau$ given in Lemma 3.2, and through the estimate on the operator $U_{s}$ given in Proposition 3.1. The estimate on the operator $U_{b}$ in the function space $N_{T}^{l}$ can be proved in the same way as in Proposition 3.1, the difference between the two spaces being only the different number of time derivatives allowed. 


\section{THE STOKES EQUATIONS: ASYMPTOTIC ANALYSIS}

In this section we shall analyze Equations (1)-(5) in the limit of small viscosity. We impose an initial condition of the form

$$
\boldsymbol{u}_{0}=\boldsymbol{u}_{0}^{\mathrm{E}}+\boldsymbol{u}_{0}^{\mathrm{P}}+\varepsilon \boldsymbol{w}_{0}
$$

with

$$
\boldsymbol{u}_{0}^{\mathrm{E}} \in H^{l}, \quad u_{0 \phi}^{\mathrm{P}} \in K^{l, \mu}, \quad \boldsymbol{w}_{0} \in L^{l},
$$

and $\boldsymbol{u}_{0}^{\mathrm{P}}=\left(\varepsilon u_{0 r}^{\mathrm{P}}, u_{0 \phi}^{\mathrm{P}}\right)$, where the radial component $u_{0 r}^{\mathrm{P}}$ is determined through the incompressibility condition, see Equation (55) later. The initial conditions satisfy the following compatibility conditions

$$
\begin{aligned}
& \gamma_{R} u_{0 r}^{\mathrm{E}}=g_{r}(t=0)=0, \\
& \gamma_{R} u_{0 \phi}^{\mathrm{P}}=-\gamma_{R} u_{0 \phi}^{\mathrm{E}}+g_{\phi}(t=0), \\
& \gamma_{R} \boldsymbol{w}_{0}=\left(-\gamma_{R} u_{0 r}^{\mathrm{P}}, 0\right) .
\end{aligned}
$$

Moreover the initial conditions satisfy the incompressibility conditions:

$$
\begin{aligned}
\boldsymbol{\nabla} \cdot \boldsymbol{u}_{0}^{\mathrm{E}} & =0, \\
u_{0 r}^{\mathrm{P}} & =\frac{1}{R+\varepsilon Y} \int_{Y}^{\infty} \mathrm{d} Y^{\prime} \partial_{\phi} u_{0 \phi}^{\mathrm{P}}, \\
\boldsymbol{\nabla} \cdot \boldsymbol{w}_{0} & =0 .
\end{aligned}
$$

We look for a solution of Equations (1)-(5) of the form:

$$
u_{r}=u_{r}^{\mathrm{E}}+\varepsilon u_{r}^{\mathrm{P}}+\varepsilon w_{r}, \quad u_{\phi}=u_{\phi}^{\mathrm{E}}+u_{\phi}^{\mathrm{P}}+\varepsilon w_{\phi}, \quad p=p^{\mathrm{E}}+\varepsilon p^{w},
$$

where $\boldsymbol{u}^{\mathrm{E}}, \boldsymbol{u}^{\mathrm{P}}$ and $\boldsymbol{w}$ are the inviscid solution, the boundary layer solution, and the correction term, respectively, and satisfy the following systems:

$$
\begin{aligned}
& \partial_{t} \boldsymbol{u}^{\mathrm{E}}+\nabla p^{\mathrm{E}}=0, \\
& \nabla \cdot \boldsymbol{u}^{\mathrm{E}}=0, \\
& \gamma_{R} u_{r}^{\mathrm{E}}=g_{r}(k, t), \\
& \boldsymbol{u}^{\mathrm{E}}(r, k, t=0)=\boldsymbol{u}_{0}^{E} . \\
& \left(\partial_{t}-\partial_{Y Y}+\frac{\varepsilon^{2} k^{2}}{R^{2}}\right) u_{\phi}^{\mathrm{P}}=0, \\
& \gamma u_{\phi}^{\mathrm{P}}=-\gamma_{R} u_{\phi}^{\mathrm{E}}+g_{\phi}, \\
& u_{\phi}^{\mathrm{P}}(Y \rightarrow \infty, \phi, t)=0, \\
& u_{\phi}^{\mathrm{P}}(Y, \phi, t=0)=u_{0 \phi}^{\mathrm{P}} .
\end{aligned}
$$




$$
\begin{aligned}
& \partial_{t} w_{\phi}-\varepsilon^{2}\left[\left(\partial_{r r}+\frac{1}{r} \partial_{r}-\frac{k^{2}}{r^{2}}\right) w_{\phi}-\frac{1}{r^{2}} w_{\phi}+\frac{2 i k}{r^{2}} w_{r}\right]+\frac{1}{r} \partial_{\phi} p^{w}=f_{\phi} \\
& \partial_{t} w_{r}-\varepsilon^{2}\left[\left(\partial_{r r}+\frac{1}{r} \partial_{r}-\frac{k^{2}}{r^{2}}\right) w_{r}-\frac{1}{r^{2}} w_{r}-\frac{2 i k}{r^{2}} w_{\phi}\right]+\partial_{r} p^{w}=f_{r} \\
& \nabla \cdot \boldsymbol{w}=0 \\
& \gamma_{R} \boldsymbol{w}=\left(-\gamma u_{r}^{\mathrm{P}}, 0\right) \\
& \boldsymbol{w}(r, k, t=0)=\boldsymbol{w}_{0}
\end{aligned}
$$

where the expressions of the source terms $\left(f_{r}(r, k, t) ; f_{\phi}(r, k, t)\right)$ are given by

$$
\begin{aligned}
f_{r}=\varepsilon & \left(-\frac{i k}{r^{2}} u_{\phi}^{\mathrm{P}}+\Delta u_{0 r}^{\mathrm{E}}-\frac{u_{0 r}^{\mathrm{E}}}{r^{2}}-\frac{2 i k}{r^{2}} u_{0 \phi}^{\mathrm{E}}\right)+\varepsilon^{2} \frac{k^{2}}{R^{2}} \frac{r^{2}-R^{2}}{r^{2}} u_{r}^{\mathrm{P}}, \\
f_{\phi}=\varepsilon & {\left[\frac{1}{r} \partial_{r} u_{\phi}^{\mathrm{P}}-\frac{u_{\phi}^{\mathrm{P}}}{r^{2}}\left(1-\frac{k^{2}}{R^{2}}\left(r^{2}-R^{2}\right)\right)+\Delta u_{0 \phi}^{\mathrm{E}}-\frac{u_{0 \phi}^{\mathrm{E}}}{r^{2}}+\frac{2 i k}{r^{2}} u_{0 r}^{\mathrm{E}}\right] } \\
& +\varepsilon^{2} \frac{2 i k}{r^{2}} u_{r}^{\mathrm{P}}
\end{aligned}
$$

The Euler equations (57)-(60) have been obtained from Equations (1)-(5) neglecting the viscosity term and imposing the boundary condition only for the radial component. Prandtl equations are obtained writing Equations (1)-(5) in terms of the rescaled radial variable $Y=(r-R) / \varepsilon$ and imposing the usual boundary layer approximation. The boundary condition (62) ensures the right value at the boundary for $\boldsymbol{u}$. The radial velocity $\varepsilon u_{r}$, which is $O(\varepsilon)$, can be computed from the incompressibility condition. Notice that in writing the expressions (70) and (71) for the source term we have used the fact that $\Delta \boldsymbol{u}^{\mathrm{E}}=\Delta \boldsymbol{u}_{0}^{\mathrm{E}}$., we shall prove this in the following subsection, see Equation (74) later.

We now solve the above equations.

\subsection{Euler Equations}

The solution of Equations (57)-(60) is given by

$$
\boldsymbol{u}^{\mathrm{E}}=\boldsymbol{u}_{0}^{\mathrm{E}}+\nabla N g_{r},
$$

where the operator $N$, defined by

$$
N=-\frac{R}{|k|}\left(\frac{R}{r}\right)^{|k|},
$$


solves the Laplace equation with Neumann boundary condition, i.e.

$$
\begin{aligned}
\Delta N g_{r} & =0, \\
\gamma_{R} \partial_{r} N g_{r} & =g_{r} .
\end{aligned}
$$

The expression of the pressure is given by

$$
p^{\mathrm{E}}=N \partial_{t} g_{r} \text {. }
$$

From Equation (72) it is clear that

$$
\Delta \boldsymbol{u}^{\mathrm{E}}=\Delta \boldsymbol{u}_{0}^{\mathrm{E}} .
$$

\subsection{Prandtl Equations}

Let us first introduce the heat operators in the half plane. The heat kernel $E_{0}(k, Y, t)$ is defined by

$$
E_{0}(k, Y, t)=\frac{1}{(4 \pi t)^{1 / 2}} \mathrm{e}^{-\frac{\varepsilon^{2} k^{2}}{R^{2}} t} \mathrm{e}^{-\frac{Y^{2}}{4 t}} .
$$

The convolution between the heat kernel and the odd extension (to $Y<0$ ) of the function $f$ is the operator $E_{0}(t)$, namely

$$
E_{0}(t) f=\int_{0}^{\infty} \mathrm{d} Y^{\prime}\left[E_{0}\left(k, Y-Y^{\prime}, t\right)-E_{0}\left(k, Y+Y^{\prime}, t\right)\right] f\left(Y^{\prime}\right) .
$$

It solves the heat equation on the half plane $Y>0$ with initial data $f$ and with zero boundary data.

The operator $E_{1}$ solves the heat equation with boundary data $g$ and zero initial data and is given by

$$
E_{1} g(t)=\int_{0}^{t} \mathrm{~d} s \mathrm{e}^{-\frac{\varepsilon^{2} k^{2}}{R^{2}}(t-s)} \frac{Y}{t-s} \frac{\mathrm{e}^{-Y^{2} / 4(t-s)}}{\sqrt{4 \pi(t-s)}} g(s) .
$$

If one introduces the operator $M$, which acts on vector functions and is defined as

$$
M g=g_{\phi}+N^{\prime} g_{r},
$$

and using Equation (72) one can write the boundary condition for $u_{\phi}^{\mathrm{P}}$, Equation (62), in the form

$$
\gamma u_{\phi}^{\mathrm{P}}=M \boldsymbol{g}-\gamma_{R} u_{0 \phi}^{\mathrm{E}} .
$$

Hence the solution of boundary layer equation (61) with the boundary conditions (75) and (63), and the initial condition (64) is given by

$$
u_{\phi}^{\mathrm{P}}=E_{0}(t) u_{0 \phi}^{\mathrm{P}}+E_{1}\left[M \boldsymbol{g}-\gamma_{r} u_{0 \phi}^{\mathrm{E}}\right] .
$$


The expression for the radial component $u_{r}^{\mathrm{P}}$ is obtained from the incompressibility condition:

$$
u_{r}^{\mathrm{P}}=\frac{1}{\varepsilon Y+R} \int_{Y}^{\infty} \mathrm{d} Y^{\prime} \partial_{\phi} u_{\phi}^{\mathrm{P}} .
$$

\subsection{The Correction Term}

Equations (65)-(69) for the correction $w$ are of the same form as Equations (30)-(34). Therefore the solution is explicitly given by Equations (43) and (44)

$$
\begin{aligned}
w_{r} & =U\left[\tau^{\prime}(r, k, t),|k| N^{\prime} \beta\right], \\
w_{\phi} & =N^{\prime}\left[\tau^{\prime}(r, k, t)-w_{r}(r, k, t)\right],
\end{aligned}
$$

where we have defined $\tau^{\prime}(r, k, t)$ as

$$
\tau^{\prime}(r, k, t)=\mathcal{E}_{k-1}^{(2)} V_{1} \boldsymbol{f}+\mathcal{E}_{k-1}^{(1)}|k| N^{\prime} \beta+\mathcal{E}_{k-1}^{(0)} V_{1} \boldsymbol{w}_{0} .
$$

In the above expression $\boldsymbol{f}$ is given by Equations (70) and (71), while $\beta$ is defined as

$$
\beta=-\frac{1}{R} \int_{0}^{\infty} u_{\phi}^{\mathrm{P}}\left(Y^{\prime}, k, t\right) \mathrm{d} Y^{\prime} .
$$

To give a better estimate on the error it is useful to decompose $V_{1} f(r, k, t)$ into the sum of two terms:

$$
V_{1} \boldsymbol{f}(r, k, t)=\mathcal{F}^{l-1}(r, k, t)+\varepsilon \mathcal{F}^{l-2}(r, k, t),
$$

where

$$
\begin{aligned}
\mathcal{F}^{l-1}(r, k, t)= & \varepsilon\left\{\frac{1}{r^{2}}\left(-i k+N^{\prime}\right) u_{\phi}^{\mathrm{P}}-\frac{N^{\prime}}{r} \partial_{r} u_{\phi}^{\mathrm{P}}-\frac{1}{r^{2}}\left(u_{0 r}^{\mathrm{E}}-N^{\prime} u_{0 \phi}^{\mathrm{E}}\right)\right. \\
& \left.-\frac{2 i k}{r^{2}}\left(u_{0 \phi}^{\mathrm{E}}+N^{\prime} u_{0 r}^{\mathrm{E}}\right)\right\}, \\
\mathcal{F}^{l-2}(r, k, t)= & N^{\prime}\left(\frac{1}{r^{2}}-\frac{1}{R^{2}}\right) k^{2} u_{\phi}^{\mathrm{P}}+\Delta\left(u_{0 r}^{\mathrm{E}}-N^{\prime} u_{0 \phi}^{\mathrm{E}}\right) \\
& +\varepsilon u_{r}^{\mathrm{P}}\left(\frac{2|k|}{r^{2}}+\frac{k^{2}}{R^{2}} \frac{r^{2}-R^{2}}{r^{2}}\right) .
\end{aligned}
$$

The reason of the above decomposition is that, as we shall see in the following section, $\mathcal{F}^{l-1}$ and $\mathcal{F}^{l-2}$ give rise to two different contributions to $\varepsilon \boldsymbol{w}$. The term originating from $\mathcal{F}^{l-1}$ will be shown to belong to $L_{T}^{l-1}$ with norm $O(\varepsilon)$. The term originating from $\mathcal{F}^{l-2}$ will have norm $O\left(\varepsilon^{2}\right)$ in $L_{T}^{l-2}$. 
The above decomposition for $V_{1} \boldsymbol{f}$ gives rise to an analogous decomposition for $\tau^{\prime}$ as given in Equation (80). In fact one can write

$$
\tau^{\prime}=\tau^{\prime l-1}+\varepsilon \tau^{\prime l-2}
$$

where

$$
\begin{aligned}
\tau^{\prime-1}(r, k, t) & =\mathcal{E}_{k-1}^{(2)} \mathcal{F}^{l-1}+\mathcal{E}_{k-1}^{(1)}|k| N^{\prime} \beta+\mathcal{E}_{k-1}^{(0)} V_{1} \boldsymbol{w}_{0} \\
\tau^{\prime l-2}(r, k, t) & =\mathcal{E}_{k-1}^{(2)} \mathcal{F}^{l-2} .
\end{aligned}
$$

The decomposition (82) for $\tau^{\prime}$ allows one to see that the correction $\boldsymbol{w}$, as given by Equations (78) and (79) has the following structure:

$$
\boldsymbol{w}=\boldsymbol{w}^{\mathrm{E}}+\boldsymbol{w}^{l-1}+\varepsilon \boldsymbol{w}^{l-2}
$$

where

$$
\begin{array}{ll}
w_{r}^{\mathrm{E}}=U_{b}\left(|k| N^{\prime} \beta\right), & w_{\phi}^{\mathrm{E}}=-N^{\prime} U_{b}\left(|k| N^{\prime} \beta\right) ; \\
w_{r}^{l-1}=U_{s}\left(\tau^{\prime l-1}\right), & w_{\phi}^{l-1}=N^{\prime}\left(\tau^{\prime l-1}-U_{s}\left(\tau^{\prime l-1}\right)\right) ; \\
w_{r}^{l-2}=U_{s}\left(\tau^{\prime l-2}\right), & w_{\phi}^{l-2}=N^{\prime}\left(\tau^{\prime l-2}-U_{s}\left(\tau^{l-2}\right)\right) .
\end{array}
$$

We remind that the operators $U_{b}$ and $U_{s}$ have been introduced in Equation (17) and Equation (18), respectively.

\subsection{Estimates}

In this section we give the estimates on the inviscid term $\boldsymbol{u}^{\mathrm{E}}$, on the boundary layer term $\boldsymbol{u}^{\mathrm{P}}$ and on the correction term $\boldsymbol{w}$. For sake of notational simplicity we introduce the norm $\|\cdot \cdot\|_{l, \mu}$. Suppose $\boldsymbol{u}_{0}$ has the structure given in Equation (49) with $\boldsymbol{u}_{0}^{\mathrm{E}} \in H^{l}, u_{0 \phi}^{\mathrm{P}} \in K^{l, \mu}$ and $\boldsymbol{w}_{0} \in L^{l}$. Then we define $\left\|\boldsymbol{u}_{0}\right\|_{l, \mu}$ as

$$
\left\|\boldsymbol { u } _ { 0 } \left|\left\|_{l, \mu}=\left|\boldsymbol{u}_{0}^{\mathrm{E}}\right|_{l}+\left|u_{0 \phi}^{\mathrm{P}}\right|_{l, \mu}+\right\| \boldsymbol{w}_{0} \|_{l} .\right.\right.
$$

We begin with the inviscid part $\boldsymbol{u}^{\mathrm{E}}$, solution of Equations (57)-(60). First we state an estimate on the operator $N$ as defined in Equation (73).

Lemma 4.1. Let $\psi \in H_{T}^{\prime l}$. Then $\nabla N \psi \in H_{T}^{l}$, and the following estimate holds:

$$
|\nabla N \psi|_{l, T} \leq c|\psi|_{l, T}
$$

The proof is obvious and is based on the representation (73) for the operator $N$ and on the fact that $(R / r)^{|k|} \leq 1$. 
Proposition 4.1. Let $\boldsymbol{g} \in H_{T}^{\prime l}$ and let $\boldsymbol{u}_{0}$ satisfy Equations (49)-(56). Then $\boldsymbol{u}^{\mathrm{E}} \in$ $H_{T}^{l}$ and the following estimate holds:

$$
\left|\boldsymbol{u}^{\mathrm{E}}\right|_{l, T} \leq c\left(\left\|\left.\left|\boldsymbol{u}_{0} \|_{l, \mu}+\right| \boldsymbol{g}\right|_{l, T}\right) .\right.
$$

The proof is based on the explicit representation (72) for $\boldsymbol{u}^{\mathrm{E}}$, and on Lemma 4.1. We now pass to the boundary layer solution $\boldsymbol{u}^{\mathrm{P}}$, solution of Equations (61)-(64).

Proposition 4.2. Let $g \in H_{T}^{\prime l}$ and let $\boldsymbol{u}_{0}$ satisfy Equations (49)-(56). Then $u_{\phi}^{\mathrm{P}} \in$ $K_{T}^{l, \mu}, u_{r}^{\mathrm{P}} \in K_{T}^{l-1, \mu}$, and the following estimate holds:

$$
\begin{aligned}
\left|u_{\phi}^{\mathrm{P}}\right|_{l, \mu, T} & \leq c\left(\left\||| \boldsymbol{u}_{0}\right\|_{l, \mu}+|\boldsymbol{g}|_{l, T}\right), \\
\left|u_{r}^{\mathrm{P}}\right|_{l-1, \mu, T} & \leq c\left(\left\|\left.\left|\boldsymbol{u}_{0} \|_{l, \mu}+\right| \boldsymbol{g}\right|_{l, T}\right) .\right.
\end{aligned}
$$

The proof of the above proposition is based on the explicit representation of $u_{\phi}^{\mathrm{P}}$ given in Equation (76), on the fact that $\gamma u_{0 \phi}^{\mathrm{P}}=\left[M g-\gamma_{R} u_{0 \phi}^{\mathrm{E}}\right]_{t=0}$ (due to the compatibility conditions), and on the usual estimates on the heat operators given, e.g., in $[3,6]$. Notice the loss of regularity (one derivative) in the radial component due to the incompressibility condition. We now pass to the correction term, solution of Equations (4.17)-(4.21). We first state the following preliminary lemmas.

Lemma 4.2. Let $\boldsymbol{g} \in H_{T}^{\prime \prime}, \boldsymbol{u}_{0}$ satisfy Equations (49)-(56), and let $\beta$ be given by Equation (81). Then $\beta \in L_{T}^{\prime l}$ and the following estimate holds:

$$
\|\beta\|_{l, T} \leq c\left(|\boldsymbol{g}|_{l, T}+\left\|\boldsymbol{u}_{0}\right\|_{l, \mu}\right) .
$$

The above lemma is obvious and is based on the fact that $u_{\phi}^{\mathrm{P}} \in K_{T}^{l, \mu}$ and therefore it is decaying (exponentially) in $Y$.

Lemma 4.3. Let $\boldsymbol{g} \in H_{T}^{\prime l}, \boldsymbol{u}_{0}$ satisfy Equations (49)-(56), and let $\tau^{\prime l-1}$ be given by Equation (83). Then $\tau^{\prime l-1} \in L_{T}^{l-1}$ and the following estimate holds:

$$
\left\|\tau^{\prime l-1}\right\|_{l-1, T} \leq c\left(|\boldsymbol{g}|_{l, T}+\|\| \boldsymbol{u}_{0} \|_{l, \mu}\right) .
$$

Lemma 4.4. Let $\boldsymbol{g} \in H_{T}^{\prime l}, \boldsymbol{u}_{0}$ satisfy Equations (49)-(56), and let $\tau^{\prime l-2}$ be given by Equation (84). Then $\tau^{\prime l-2} \in L_{T}^{l-2}$ and the following estimate holds:

$$
\left\|\tau^{\prime l-2}\right\|_{l-2, T} \leq c\left(|\boldsymbol{g}|_{l, T}+\|\| \boldsymbol{u}_{0} \|_{l, \mu}\right) .
$$

The proof of Lemma 4.3 is based on the fact that $\mathcal{F}^{l-1} \in L_{T}^{l-1}$, as can be seen by a direct inspection of Equation (82), on the fact that $\gamma_{R} V_{1} \boldsymbol{w}_{0}=\left.N^{\prime} \beta\right|_{t}=0$ (compatibility conditions), and on Proposition 3.2. The proof of Lemma 4.4 is 
based on the fact that $\mathcal{F}^{l-2} \in L_{T}^{l-2}$, as can be seen by a direct inspection of Equation (82).

With the above lemmas the following estimate on the correction $\boldsymbol{w}$ is obvious.

Proposition 4.3. Let $\boldsymbol{g} \in H_{T}^{\prime l}$ and let $\boldsymbol{u}_{0}$ satisfy Equations (49)-(56). Then $\boldsymbol{w}$ can be decomposed as in Equation (85), with $\boldsymbol{w}^{\mathrm{E}} \in N_{T}^{l-1}, \boldsymbol{w}^{l-1} \in L_{T}^{l-1}, \boldsymbol{w}^{l-2} \in L_{T}^{l-2}$, and the following estimates hold:

$$
\begin{aligned}
\left\|\boldsymbol{w}^{\mathrm{E}}\right\|_{l-1, T}^{N} & \leq c\left(|\boldsymbol{g}|_{l, T}+\left\|\boldsymbol{u}_{0}\right\|_{l, \mu}\right), \\
\left\|\boldsymbol{w}^{l-1}\right\|_{l-1, T} & \leq c\left(|\boldsymbol{g}|_{l, T}+\left\|\boldsymbol{u}_{0}\right\|_{l, \mu}\right), \\
\left\|\boldsymbol{w}^{l-2}\right\|_{l-2, T} & \leq c\left(|\boldsymbol{g}|_{l, T}+\left\|\boldsymbol{u}_{0}\right\|_{l, \mu}\right) .
\end{aligned}
$$

\subsection{The Main Result}

We summarize our results in the following theorem.

Theorem 4.1. Let $\boldsymbol{g} \in H_{T}^{\prime l}$ and let $\boldsymbol{u}_{0}$ satisfy Equations (49)-(56). Then the solution of Equations (1)-(5) is of the form:

$$
\begin{aligned}
u_{r}= & u_{r}^{\mathrm{E}}+\varepsilon u_{r}^{\mathrm{P}}+\varepsilon w_{r}^{\mathrm{E}}+\varepsilon w_{r}^{l-1}+\varepsilon^{2} w_{r}^{l-2}, \quad u_{\phi}=u_{\phi}^{\mathrm{E}}+u_{\phi}^{\mathrm{P}} \\
& +\varepsilon w_{\phi}^{\mathrm{E}}+\varepsilon w_{\phi}^{l-1}+\varepsilon^{2} w_{\phi}^{l-2}, \quad p=p^{\mathrm{E}}+\varepsilon p^{w},
\end{aligned}
$$

where $\boldsymbol{u}^{\mathrm{E}} \in H_{T}^{l}, u_{\phi}^{\mathrm{P}} \in K^{l, \mu, T}, u_{r}^{\mathrm{P}} \in K^{l-1, \mu, T}, \boldsymbol{w}^{\mathrm{E}} \in N_{T}^{l-1}, \boldsymbol{w}^{l-1} \in L_{T}^{l-1}, \boldsymbol{w}^{l-2} \in$ $L_{T}^{l-2}$. The following estimates hold:

$$
\begin{aligned}
\left|\boldsymbol{u}^{\mathrm{E}}\right|_{l, T} & \leq c\left(|\boldsymbol{g}|_{l, T}+\left\|\mid \boldsymbol{u}_{0}\right\|_{l, \mu}\right), \\
\left|u_{\phi}^{\mathrm{P}}\right|_{l, \mu, T} & \leq c\left(|\boldsymbol{g}|_{l, T}+\left\|\mid \boldsymbol{u}_{0}\right\|_{l, \mu}\right), \\
\left|u_{r}^{\mathrm{P}}\right|_{l-1, \mu, T} & \leq c\left(|\boldsymbol{g}|_{l, T}+\left\|\mid \boldsymbol{u}_{0}\right\|_{l, \mu}\right), \\
\left\|\boldsymbol{w}^{\mathrm{E}}\right\|_{l-1, T}^{N} & \leq c\left(|\boldsymbol{g}|_{l, T}+\left\|\mid \boldsymbol{u}_{0}\right\|_{l, \mu}\right), \\
\left\|\boldsymbol{w}^{l-1}\right\|_{l-1, T} & \leq c\left(|\boldsymbol{g}|_{l, T}+\left\|\mid \boldsymbol{u}_{0}\right\|_{l, \mu}\right), \\
\left\|\boldsymbol{w}^{l-2}\right\|_{l-2, T} & \leq c\left(|\boldsymbol{g}|_{l, T}+\left\|\mid \boldsymbol{u}_{0}\right\|_{l, \mu}\right) .
\end{aligned}
$$

\section{CONCLUDING REMARKS}

In this paper we have considered the zero viscosity limit of the time-dependent Stokes equations in the exterior of a disk. We proved that away from the boundary the solution of the Stokes equations converges to the solution of the linearized Euler equations. Close to the boundary instead, the solution has the structure of a boundary layer whose size is the square root of the viscosity. Several related 
problems suggest themselves. One could ask if the same results apply in the case of the full Navier-Stokes equations. In this case we believe that the additional hypothesis of analyticity of the initial data is necessary to prevent the appearance of a singularity in the solution of Prandtl equations. Also interesting would be the analysis of the Navier-Stokes equations linearized around a background flow (Oseen equations). In fact the presence of an inflection point in the background flow, with the appearance of instability, could destroy the regular asymptotic structure of the Stokes equations. These topics are under current investigations, and will be the subject of a forthcoming paper.

\section{ACKNOWLEDGMENTS}

The first and third authors were supported in part by MURST under grant $40 \%$, and the second author was supported by the Army Research Office under grant \#DAAG-98-1-0323.

\section{REFERENCES}

1. Sammartino, M.; Caflisch, R.E. Zero Viscosity Limit for Analytic Solutions of the Navier-Stokes Equation on a Half-Space II. Construction of the NavierStokes Solution. Comm. Math. Phys. 1998, 192, 463-491.

2. Ukai, S. A Solution Formula for the Stokes Equation in $R_{+}^{n}$. Comm. Pure Appl. Math. 1987, 40, 611-621.

3. Ladyzenskaja, O.A.; Solonnikov, V.A.; Uralćeva, N.N. Linear and Quasilinear Equations of Parabolic Type; Transl. Math. Monographs; AMS: Providence, RI; Vol. 23, 1991.

4. Sammartino, M. The Boundary Layer Analysis for Stokes Equations on a Half Space. Comm. Part. Diff. Eqs. 1997, 22(5/6), 749-771.

5. Davies, B. Integral Transforms and Their Applications; Springer-Verlag: Berlin 1985.

6. Sammartino, M.; Caflisch, R.E. Zero Viscosity Limit for Analytic Solutions of the Navier-Stokes Equation on a Half-Space I. Existence for Euler and Prandtl Equations. Comm. Math. Phys. 1998, 192, 433-461.

Received October 1999

Revised August 2000 


\section{Request Permission or Order Reprints Instantly!}

Interested in copying and sharing this article? In most cases, U.S. Copyright Law requires that you get permission from the article's rightsholder before using copyrighted content.

All information and materials found in this article, including but not limited to text, trademarks, patents, logos, graphics and images (the "Materials"), are the copyrighted works and other forms of intellectual property of Marcel Dekker, Inc., or its licensors. All rights not expressly granted are reserved.

Get permission to lawfully reproduce and distribute the Materials or order reprints quickly and painlessly. Simply click on the "Request

Permission/Reprints Here" link below and follow the instructions. Visit the U.S. Copyright Office for information on Fair Use limitations of U.S. copyright law. Please refer to The Association of American Publishers' (AAP) website for guidelines on Fair Use in the Classroom.

The Materials are for your personal use only and cannot be reformatted, reposted, resold or distributed by electronic means or otherwise without permission from Marcel Dekker, Inc. Marcel Dekker, Inc. grants you the limited right to display the Materials only on your personal computer or personal wireless device, and to copy and download single copies of such Materials provided that any copyright, trademark or other notice appearing on such Materials is also retained by, displayed, copied or downloaded as part of the Materials and is not removed or obscured, and provided you do not edit, modify, alter or enhance the Materials. Please refer to our Website User Agreement for more details.

\section{Order now!}

Reprints of this article can also be ordered at http://www.dekker.com/servlet/product/DOI/101081PDE100001758 